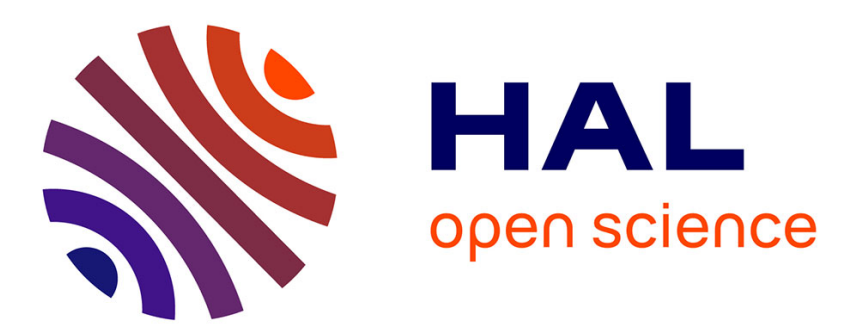

\title{
Average unit-cell approach to diffraction on Thue-Morse sequence and decorated quasicrystals
}

Janusz Wolny, Anna Wnęk, Jean-Louis Verger-Gaugry, Lucjan Pytlik

\section{To cite this version:}

Janusz Wolny, Anna Wnęk, Jean-Louis Verger-Gaugry, Lucjan Pytlik. Average unit-cell approach to diffraction on Thue-Morse sequence and decorated quasicrystals. Materials Science and Engineering: A, 2000, 294-296, pp.381-384. 10.1016/S0921-5093(00)01154-0 . hal-03135113

\section{HAL Id: hal-03135113 \\ https://hal.science/hal-03135113}

Submitted on 9 Feb 2021

HAL is a multi-disciplinary open access archive for the deposit and dissemination of scientific research documents, whether they are published or not. The documents may come from teaching and research institutions in France or abroad, or from public or private research centers.
L'archive ouverte pluridisciplinaire HAL, est destinée au dépôt et à la diffusion de documents scientifiques de niveau recherche, publiés ou non, émanant des établissements d'enseignement et de recherche français ou étrangers, des laboratoires publics ou privés. 


\title{
Average unit-cell approach to diffraction on ThueMorse sequence and decorated quasicrystals
}

\author{
Janusz, Wolny ${ }^{\ddagger}$, Anna Wnek ${ }^{\ddagger}$, Jean-Louis Verger-Gaugry ${ }^{\dagger}$ \\ and Lucjan Pytlik \\ ¥ AGH UST - Faculty of Physics and Nuclear Techniques, University of Mining and \\ Metallurgy, al. Mickiewicza 30, 30-059 Krakow, Poland. \\ $\dagger$ Institut Fourier, University Joseph Fourier Grenoble, CNRS URA 188, BP 74- Do- \\ maine Universitaire, 38402- Saint Martin dHères, France.
}

\begin{abstract}
The average unit cell approach has been successfully used to construct the probability distributions of atomic displacements from the reference lattice points and to calculate the diffraction patterns for two aperiodic structures the ThueMorse sequence and decorated Fibonacci chains. For the singular continuous diffraction pattern of the ThueMorse sequence fractal exponents and multiplication factors have been found for scaling with the number of atoms. General method able to distinguish between various quasilattice decorations has been elaborated and tested for decorated Fibonacci chains. .
\end{abstract}




\section{Introduction}

Recently, the concept of average unit cell has been successfully used in the diffraction analysis of aperiodic structures and quasicrystals [1]. In such an approach for monoatomic structures, the structure factor for reflections indexed by main wave vector $n_{1} k$ and auxiliary (modulation) wave vector $n_{2} q$ can be written as follows:

$F=N f \int_{-u_{1}}^{u_{1}} \int_{-v_{1}}^{v_{1}} P_{k, q}(u, v) \exp \left[i\left(n_{1} k u+n_{2} q v\right)\right] \mathrm{d} u \mathrm{~d} v$

$n_{1}, n_{2} \in \mathbf{Z}, u_{1}=\pi / k, v_{1}=\pi / q$, where $P_{k, q}(u, v)$ is the probability distribution of atomic displacements from the reference lattice points defined in [1]. The same distribution $P_{k, q}(u, v)$ describes the average unit cell for the whole series of main reflections $\left(n_{1} k\right)$ and their satellites $\left(n_{2} q\right)$. In order to calculate the diffraction peak intensities, one has to know the respective probability distribution and in this paper such distributions have been obtained for two types of structures: the Thue-Morse sequence and the decorated Fibonacci chain. For the Thue-Morse sequence the satellite peak intensities scale fractally with the number of atoms. However, it is possible to parameterise the distributions by especially defined $\Delta$ parameters, for which integer number calculations are possible for a wide range of cluster sizes (atom numbers in the chain).

\section{Thue-Morse sequence}

The one-side Thue-Morse sequence can be defined on a two-letter alphabet $\{0,1\}$, using the following substitution rule $\sigma: \sigma(0)=01, \sigma(1)=10[1-6]$. For such a sequence the two elements are replaced by the corresponding two bonds ( $a$ and $b$ ), and in this paper it is assumed that $b=0.1$ and $a=10 b=1[2,3,6-8]$. The numbers of those two bonds are equal, and a periodic subset, made of every second position can be selected, with a periodicity constant equal to $\lambda_{0}=(a+b)=1.1$. The corresponding wave vector is then equal to $k_{0}=2 \pi /(a+b) \approx 5.71$. The unit cell of such a superstructure is decorated by two atoms, one located at position 0 and the other one occupying aperiodically the two possible positions at the distance $a$ or $b$ from the first atom. For an infinite cluster, occupation probabilities are equal $\left(P_{a}=P_{b}=1 / 4\right)$, which leads to the expression for the Bragg reflections $\left(k=n \cdot k_{0}\right)$ given by [9-12]

$$
\frac{I(k)}{N^{2}}=\frac{1}{4}[1+\cos (k b)]^{2}
$$

\subsection{Satellite reflections}

It is known that there is a cumulation of diffuse scattering around $q_{m}=k_{0} / m\left(m \equiv 2 j+1=3,5,7, \ldots, m, j \in Z_{+}\right)$. Intensities at the satellite positions scale fractally, disappearing gradually from the diffraction pattem with increasing number of atoms. Using two reference lattices, one with 


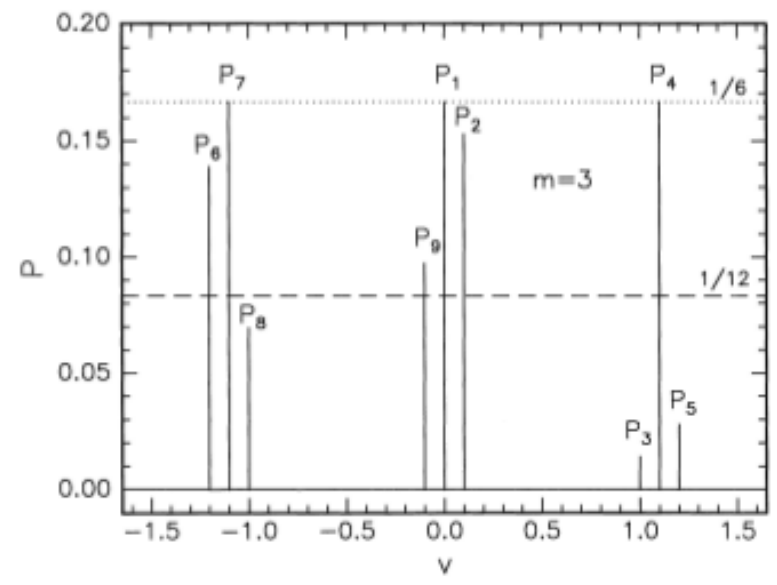

Fig. 1. Probability distribution vs. $v$ for $q_{3}$ and the number of atoms $=72$. Peaks marked as $P_{1}, P_{4}$ and $P_{7}$ are equal to $1 / 6$ and correspond to periodic positions of the superstructure. Other probabilities scale with the number of atoms and approach $1 / 12$ for the infinite cluster.

periodicity $\lambda_{0}=(a+b)=1.1$ (corresponding to $\left.k_{0}\right)$ and the other one with periodicity equal to $3 \lambda_{0}$ (which corresponds to the scattering vector $q_{3}=k_{0} / 3$ ( $m=3$ ) describing positions of an appropriate satellite), one gets the probability distribution shown in Fig. 1. The probability distribution in such an average unit cell is non-zero for nine positions only, and, for the number of atoms given by $N=6 n, n \in \mathbf{Z}_{+}$, the distribution values are related as follows:

$P_{1}=P_{4}=P_{7}=P_{2}+P_{3}=P_{5}+P_{6}=P_{8}+P_{9}=1 / 6$

A set of three parameters $\Delta_{i}(i=1,2,3)$ can be defined, such that

$\Delta_{1}=\left(P_{2}-P_{3}\right) N ; \quad \Delta_{2}=\left(P_{6}-P_{5}\right) N ;$

$\Delta_{3}=\left(P_{9}-P_{8}\right) N$

The structure factor is then given by $\left(k=n_{1} k_{0}, q=n_{2} q_{3}\right)$

$F(k+q)=F_{1}(k+q)+F_{2}(k+q)$

where

$$
\begin{aligned}
F_{1}= & \frac{N}{6}\{1+2 \cos (q(a+b))+\cos ((k+q) b) \\
& +\cos (k b-q a)+\cos (k b+q(a+2 b))\} \\
F_{2}= & \frac{\Delta_{1}}{2}[\exp (i(k+q) b)-\exp (i(q a-k b))] \\
& -i \Delta_{2} \sin (k b+q(a+2 b))-\frac{\Delta_{3}}{2}[\exp (i(k b-q a)) \\
& -\exp (-i(k+q) b)]
\end{aligned}
$$

The first part of the structure factor $\left(F_{1}\right)$ leads, for $q=0$, to the expression (2) for the diffraction intensity of Bragg reflections. For other satellites described by $q_{m}=k_{0} / m$, where $m=5$ or 7 , five and seven parameters $\Delta_{i}$ can be defined

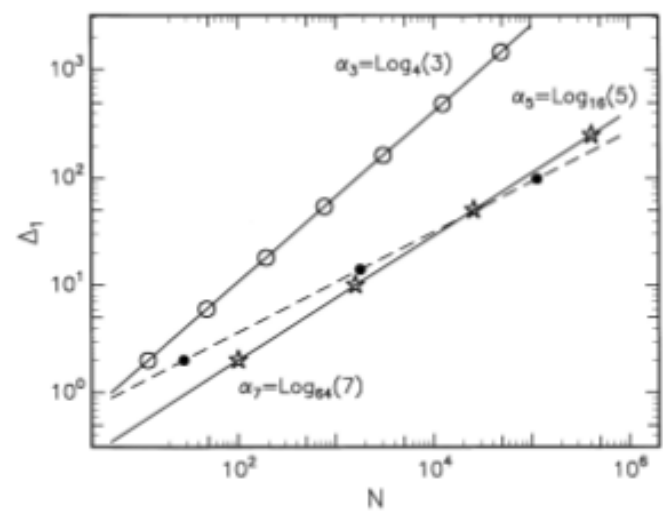

Fig. 2. Double logarithmic plots of $\Delta_{1}$, for $m=3,5,7$, vs. the selected number of atoms. Solid lines represent fractal scaling and the corresponding exponents $\alpha$ are given in the figure.

respectively in a similar way as before, and they all depend on the number of atoms. From our integer calculations it was found that there are multiplication factors equal to

$d_{m}=2^{2 j}=\{4,16,64, \ldots\}$,

for $m=2 j+1=\{3,5,7 \ldots\}$, respectively, such that all the $\Delta_{i}$ behave similarly after multiplication of the number of atoms $(N)$ by the appropriate $d_{m}$ factor. In Fig. 2, the $\Delta_{1}$ parameters for various satellites have been plotted versus the number of atoms. In a double logarithmic plots, stric tly valid linear behaviour is observed, which means that $\Delta_{i} \sim N^{\alpha}$, where for each $m$ th satellite, the fractal exponent $\alpha^{m}$ is equal to

$\alpha_{m}=\log _{d_{m}}(m)=\frac{1}{2 j} \log _{2}(2 j+1)$

It has been checked numerically that for any $m$ th satellite and the number of atoms given by $N=4 m n, n \in \mathbf{Z}_{+}$, all the $\Delta_{i} / N^{\mathrm{a}}$ are periodic functions of $\log (N)$, with periodicity constant equal to $\log \left(d_{m}\right)=2 j \log (2)$. This allows drawing a plot of $\Delta_{i} / N^{a}$ versus $\xi$

$\xi_{m} \equiv\left\{\log _{d}(N) \bmod 1\right\}=\left\{\frac{1}{2 j} \log _{2}(N) \bmod 1\right\}$

which is shown in Fig. 3 for $m=3$. For each $\Delta$, a common curve is obtained, which is independent of the number of atoms. Actually, the curves look rather complex, and a more general expression cannot be given at the moment. However, we have found the exact values for some special points and the results will be discussed separately [12].

For all numbers of atoms equal to odd multiples of $4 \mathrm{~m}$, the positions of points, for each $\Delta_{i}$, differ from the common curves reached for the number of atoms going to infinity. However, such differences decrease rapidly to zero as $N^{-1}$. One can conclude that for large enough number of atoms (i.e. for more than a few hundred atoms), there are almost no noticeable differences between the diffraction patterns 


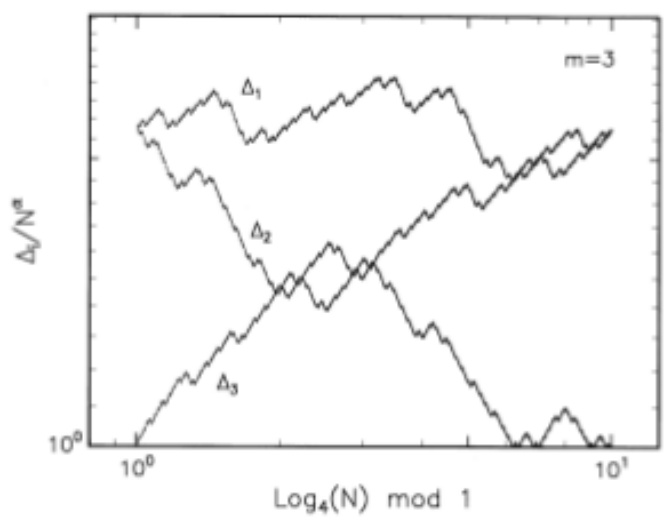

Fig. 3. Plots of $\Delta_{i} / N^{\alpha}(i=1,2,3)$ vs. $\left\{\log _{4}(N) \bmod 1\right\}$ for $q_{3}$-satellite and the number of atoms equal to even multiples of six.

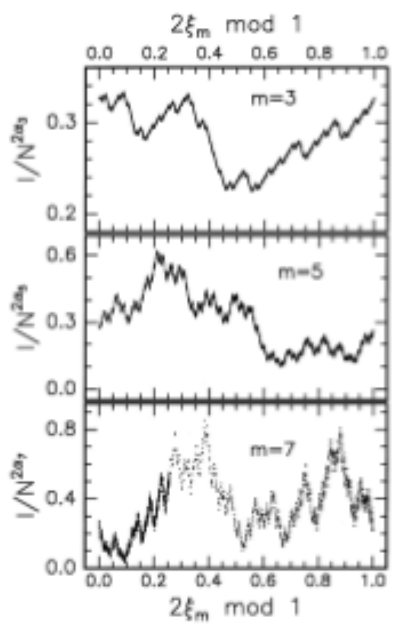

Fig. 4. Diffraction intensity for the Thue-Morse sequence at $q_{w}$-satellite positions ( $m=3,5,7)$, vs. the reduced number of atoms.

obtained for the number of atoms equal to the neighbouring numbers given by even and odd multiples of $4 \mathrm{~m}$.

The diffraction intensities at three satellite positions for scattering vector equal to $4 k_{0}+q_{m}(m=3,5,7)$ were calculated and plotted versus the number of atoms. After dividing by factor $N^{2 \alpha}$ the intensities become periodic on $\log (N)$, with periodicity constants equal to $\{2,4,16\}$, i.e. $2^{j}$, respectively. Such a scaling property leads to universal curves for the satellite intensities $\left(I / N^{2 \alpha}\right.$ versus $\left.\left\{2 \xi_{m} \bmod 1\right\}\right)$, which are shown in Fig. 4.

\section{Decorated Fibonacci chain}

Another example of application of average unit cell approach is a decorated Fibonacci chain. For value of the scattering vector corresponding to the mean distance between the atoms (i.e. $a=1+1 / \tau^{2}$, where $\tau \approx 1.618$ is the golden mean value), and a modulation vector being $\tau$-times shorter, the

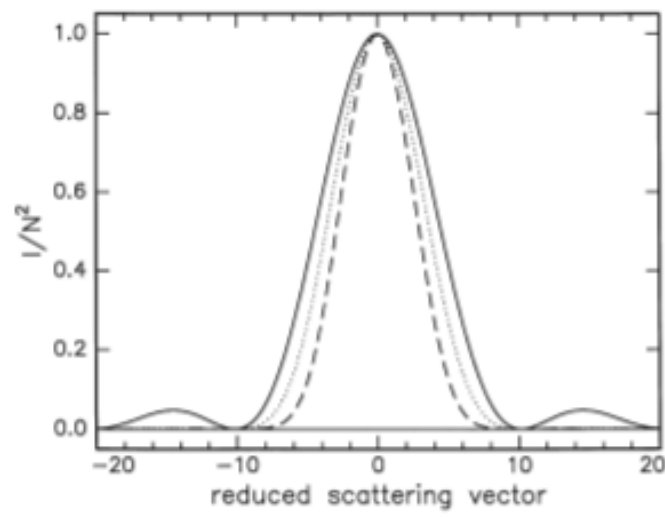

Fig. 5. Envelope functions calculated for the different quasicrystalline lattices: undecorated Fibonacci chain (solid line) and decorated Fibonacci chains with two decorations: L $\rightarrow$ LSLL, S $\rightarrow$ SLS (dotted line) and $\mathrm{L} \rightarrow \mathrm{SLL}, \mathrm{S} \rightarrow \mathrm{SSL}$ (dashed line).

calculated displacement probability distribution is non-zero only along the line given by: $v=-\tau^{2} u$. Along this line, the distribution has a rectangular shape [13] and the Fourier transform of such a distribution is the following:

$\frac{I}{N^{2}}=\left[\frac{\sin (w)}{w}\right]^{2}$

where

$w=\frac{n_{1} k-\tau^{2} n_{2} q}{2 \tau}$

The diffraction pattern consists of main reflections and their satellites, which all scale as $N^{2}$. All the peaks are described by the same envelope function shifted for different orders of the satellites. In Fig. 5 the already known results, for the decoration given by L $\rightarrow$ LSLL and S $\rightarrow$ SLS [13-15], are compared with the new non-Fibonacci type of decoration $(\mathrm{L} \rightarrow \mathrm{SLLL}$ and $\mathrm{S} \rightarrow \mathrm{SSL}$ ). In this figure, three such envelope functions have been compared for decorated and undecorated Fibonacci chains. These envelope functions can be directly determined in the diffraction experiment and they distinguish various decorations of the chain. The envelope function for the reduced scattering vector consists of many (theoretically infinitely many) reflections, so it can be obtained in a much more accurate way than the intensity of individual diffraction peaks.

\section{Conclusions}

Average-unit-cell approach was used for systematic analysis of the diffraction pattem of two different types of structures: the Thue-Morse sequence with a singular continuous diffraction pattern, and the decorated Fibonacci chain with a quasicrystalline diffraction pattem. In both cases, probability distributions of displacements from the reference lattice points were obtained and used for diffraction pattem calculations. 
For satellite reflections of Thue-Morse sequence, many properties of fractal behaviour were described for the first three type of satellites, namely for $1 / 3,1 / 5$ and $1 / 7$ satellite's indices. Fractal exponents and scaling factors were obtained for singular continuous behaviour of their diffraction intensities versus the number of atoms. General curves for scaling of satellite peak versus the number of atoms were obtained and tested for number of atoms reaching $2^{20}$.

For Fibonacci chains, the shape of the probability distribution depends directly on the decoration: it is rectangular for an undecorated chain and step-like functions are obtained for the decorated chains. The distribution is directly related to the perp-space window function known in the hyperspace description of the quasicrystals. For quasiperiodic lattices, after some additional transformation of the scattering vectors, all the diffraction peaks maxima can be described by a single envelope function which is characteristic for a given type of decoration. For the distribution shapes mentioned above the formula for the envelope function can be calculated analytically. It has been also shown that knowing several diffraction intensities, in a limited range of the scattering vector, one can successfully reconstruct the envelope function and obtain a tool able to distinguish between different decorated quasicrystalline structures.

\section{Acknowledgements}

Financial support from the Polish Committee for Scientific Research under Grant no. 2 P03B 04116 is acknowledged.

\section{References}

[1] J. Wolny, Philos. Mag. A 77 (1998) 395-412.

[2] J.-P. Allouche, M. Mendes-France, Automata and automatic sequences, in: F. Axel, D. Gratias (Eds.), Beyond Quasicrystals, Les Editions de Physique, Springer, Berlin, 1995, pp. 293-367.

[3] M. Queffelec, Dynamical systems - spectral analysis, in: Lecture Notes in Mathematics, Wol. 1294, Springer, Berlin, 1987.

[4] J.M. Luck, Phys. Rev. B 39 (1989) 5834-5849.

[5] F. Axel, J.-P. Allouche, M. Kléman, M. Mendes-France, J. Phys. (Paris) Colloq. 47 (1996) C3-181.

[6] M. Kolar, B. Jochum, L. Raymond, J. Phys. A: Math Gen. 26 (1993) 7343-7366.

[7] J.M. Luck, C. Godreche, A. Janner, T. Janssen, J. Phys A 26 (1993) 1951.

[8] F. Gähler, R. Klitzing, The diffraction pattern of self-similar tilings, in: R.V. Moody (Ed.), The Mathematics of Long-Range Aperiodic Order, NATO series, Kluwer Academic Publishers, Dordrecht, 1997, pp. 141-174.

[9] J. Wolny, Spatial fluctuations and their influence on singular diffraction pattem, in: S. Takeuchi, T. Fujiwara (Eds.), Proceedings of the Sixth Intemational Conference on Quasicrystals, World Scientific, 1998, pp. 164-167.

[10] J.-L. Verger-Gaugry, J. Wolny, J. Phys. A: Math. Gen. 32 (1999) $6445-6460$.

[11] J.-L.Verger-Gaugry, J. Wolny, J. Patera, Mathematical quasicrystals with toric interial spaces, diffraction and Thue-Morse sequence, 1999, (preprint).

[12] J. Wolny, A. Wnęk, J.-L. Verger-Gaugry, J. Computational Phys. 163 (2000) in press.

[13] Wolny, L. Pytlik, Acta Crystall. A 56 (2000) 11-19.

[14] F. Lancon, L. Billard, S. Burkov, M. de Boissieu, J. Phys (France) 4 (1994) 283-301.

[15] A. Cervellino, T. Haibach, W. Steurer, Phys. Rev. B. 57 (1998) $11223-11231$. 